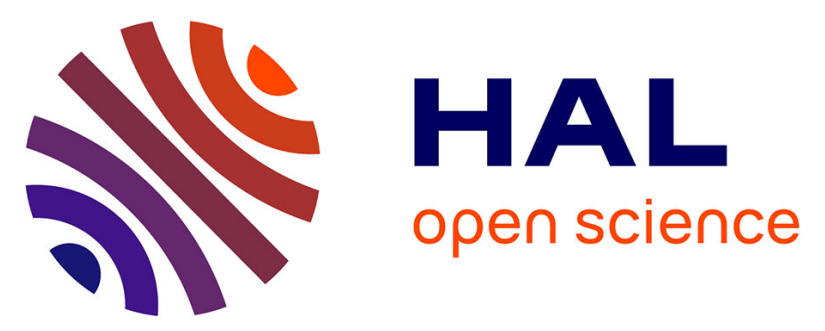

\title{
Cavitation characterization using wide band signals in an acoustic active sensing system
}

\author{
Angela Digulescu, Cornel Ioana, Florentina Bunea, Adrian Nedelcu, Ion \\ Candel, Gabriel Vasile, Alexandru Serbanescu
}

\section{- To cite this version:}

Angela Digulescu, Cornel Ioana, Florentina Bunea, Adrian Nedelcu, Ion Candel, et al.. Cavitation characterization using wide band signals in an acoustic active sensing system. OCEANS 2016 - OCEANS '16 MTS/IEEE. The Sea's the Limit, Sep 2016, Monterey, United States. pp.1 - 5, 10.1109/OCEANS.2016.7761114 . hal-01448211

\section{HAL Id: hal-01448211 \\ https://hal.science/hal-01448211}

Submitted on 27 Jan 2017

HAL is a multi-disciplinary open access archive for the deposit and dissemination of scientific research documents, whether they are published or not. The documents may come from teaching and research institutions in France or abroad, or from public or private research centers.
L'archive ouverte pluridisciplinaire HAL, est destinée au dépôt et à la diffusion de documents scientifiques de niveau recherche, publiés ou non, émanant des établissements d'enseignement et de recherche français ou étrangers, des laboratoires publics ou privés. 


\title{
Cavitation characterization using wide band signals in an acoustic active sensing system
}

\author{
Angela Digulescu ${ }^{1,2}$, Cornel Ioana ${ }^{1}$, Florentina Bunea ${ }^{3}$, Adrian Nedelcu ${ }^{3}$, Ion Candel ${ }^{1}$, Gabriel Vasile ${ }^{1}$ and Alexandru \\ Serbanescu $^{2}$ \\ ${ }^{1}$ University of Grenoble-Alpes, GIPSA-lab, Saint Martin D’Hères, France \\ ${ }^{2}$ Military Technical Academy, Bucharest, Romania \\ ${ }^{3}$ National Institute for R\&D in Electrical Engineering ICPE-CA, Bucharest, Romania \\ [angela.digulescu, cornel.ioana]@gipsa-lab.grenoble-inp.fr
}

\begin{abstract}
The paper presents a different approach based on an active acoustic system for the characterization of a vortex generating cavitation phenomenon. In the present work, the experiment was carried out in a reduced scale facility. The flow rate of the hydraulic system was varied and, when the flow became stable, the acoustic active system was started. The system is based on two ultrasonic transceivers which are placed in a "V" configuration. The main objective is to highlight the vortex presence influence on the acoustic sensing system using wide band signals. The Recurrence Plot Analysis (RPA) is used in order to highlight the vortex spectral signature, information which is not available when analyzing directly the acquired signal. The tests were done step by step, from no vortex flow to vortex flow.
\end{abstract}

Keywords- phase space, distance matrix, diagonal lines, cavitation flow

\section{INTRODUCTION}

The cavitation phenomena, widely encountered in various underwater applications, require appropriate tools for detection and analysis. Its detection and characterization is very important in terms of turbine monitoring, characterization of natural phenomena, etc. The effect of the vortex consists in the variation of the pressure in time and, in the case of a sensing system, it produces the changes of the amplitude and of the frequency - Doppler effect [1].

This paper proposes a sensing method for the cavitation characterization based on wide band acoustic signals and appropriate signals processing techniques. Our main objective is to emphasize the presence of the cavitating vortex and to characterize its stages of evolution.

The experimental data was acquired in different steady flow regimes (from no cavitation vortex to fully developed cavitation vortex). The non-intrusive technique consists in placing the ultrasound transducers in "V" configuration along the zone just above the cavitating area. The active part of the systems generates linear frequency modulation wide band signals which record on their propagation path the encountered phenomena: dispersion, noise, attenuation, but also the Doppler effect induced by the particle motion from the cavitating vortex.
The analysis of the spectral content induced by the Doppler effect in the Fourier transform is not straightforward since the Doppler spectral component are quite weak, almost invisible. For this reason, the signal analysis part is based on the Recurrence Plot Analysis method (RPA) in order to highlight the presence of the cavitation and its evolution based on the frequency content in terms of recurrence analysis [2,3].

The paper is organized as follows. In section 2, the relation between the cavitation vortex and the spectral content is emphasized. In this regard, the Doppler effect is related to the velocity distribution of the Rankine vortex [4]. Section 3 presents the concept of RPA and relates the method to the signal processing by exploiting the content of the diagonal lines of the distance matrix and their spectral content. Section 4 describes the experiment and discusses the obtained results. Then, section 5 presents the conclusions and perspectives of our work.

\section{CAVitation Vortex Flow And Its Spectral CONTENT}

Considering the velocity of the fluid described by the Navier-Stokes (1) and continuity equations (2) [4, 5]:

$$
\begin{gathered}
\frac{\partial \mathbf{u}}{\partial t}+\mathbf{u} \cdot \nabla \mathbf{u}=-\frac{1}{\rho} \nabla p+u \nabla^{2} \mathbf{u} \\
\nabla \cdot \mathbf{u}=0
\end{gathered}
$$

where $v$ is the kinematic viscosity, $p$ is the fluid pressure, $\rho$ is the density of the fluid and $\mathbf{u}$ is the velocity of the fluid and $\nabla$ is the del operator.

In the analysis of the cavitational vortex, the Rankine model is used. The Rankine vortex is described by eq. (3) [1].

$$
u_{r}=0 ; u_{z}=0 ; u_{\theta}=\left\{\begin{array}{l}
\frac{r}{r_{\max }} v_{\max }, r \leq r_{\max } \\
\frac{r_{\max }}{r} v_{\max }, r>r_{\max }
\end{array}\right.
$$

where $u_{r}, u_{z}$ and $u_{\theta}$ are the radial, axial and, respectively, tangential velocity components defined in a cylindrical coordinate system for the flow velocity, $\mathbf{u}$. As the equation 
(3) shows, when $r \leq r_{\max }$, the tangential velocity $u_{\theta}$ evolves in the forced region and, for $r>r_{\max }$, it progresses in the free vortex region.

The solution from (3) of eq. (1) and (2) is given by the frequency signature, $\omega$. Therefore, the relation between the cavitational vortex flow and the spectral content is:

$$
u_{r}=0 ; u_{z}=0 ; u_{\theta}=\left\{\begin{array}{l}
\omega \cdot r, r \leq r_{\max } \\
\frac{\omega \cdot r_{\max }^{2}}{r}, r>r_{\max }
\end{array}\right.
$$

More details about the spectral magnitude of the Doppler velocity can be found in [1].

\section{RECURRENCE PLOT ANALYSIS}

The concept of RPA is used to characterize dynamic systems in order to emphasize nonlinearities which are hard to detect otherwise. Starting from a measured time series:

$$
\mathbf{x}=\{x[1], x[2], \ldots, x[N]\}
$$

the phase space vectors are computed:

$$
\overrightarrow{v_{i}}=\sum_{k=1}^{m} x[i+(k-1) d] \cdot \overrightarrow{e_{k}}, i=\overline{1, M}
$$

where $x[\cdot]$ is the sample of the measured time series, $\overrightarrow{e_{k}}$ are the unit vectors of the phace space, $M=N-(m-1) d, d$ is the delay parameter and $m$ is the embedding dimension of the phase space. The choice of the parameters $m$ and $d$ is based on the false nearest neighbor method, respectively, on the mutual information method. More details about this method can be found in $[6,7]$.

Then, the distance/recurrence matrix is determined based on the trajectory described by the vectors from (6) by applying a point wise of distance between the points of the trajectory:

$$
\begin{aligned}
& D(i, j)=\Delta\left(v_{i}, v_{j}\right) \\
& R(i, j)=\Theta\left(\varepsilon-\Delta\left(v_{i}, v_{j}\right)\right)
\end{aligned}
$$

In eq. (7), $D(i, j)$ and $R(i, j)$ are the elements of the distance matrix, respectively recurrence matrix which correspond to the points $i$ and $j$ from the phase space. Also, $\Delta\left(v_{i}, v_{j}\right)$ is the type of distance applied between the points (Euclidean distance, norm L1, norm infinity, angular distance [6], etc.), $\varepsilon$ is the threshold (generally constant) that determines whether two points $i$ and $j$ are recurrent and $\Theta$ is the Heaviside step function. Fig. 1 illustrates the method.

The main difference between the distance matrix and the recurrence matrix is that, the first one is represented on a continuous colored scale, whereas the latter is binary plotted depending on the value of the threshold $\varepsilon$.

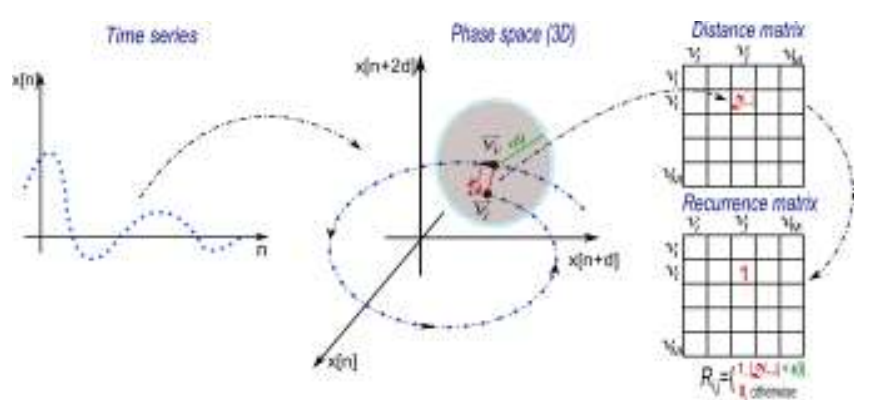

Fig. 1: The RPA concept

The quality of the trajectory which determines the recurrence/distance matrix is influenced by the delay parameter, $d$ [7]. When its value is too small, then the trajectory is overly folded around the first bisector of the phase space (redundancy case), but if its value is too large, then the trajectory becomes too complicated (irrelevance case).

Regarding the embedding dimension, $m$, it must be emphasized that if it is smaller than the real representation dimension, then the result is just a projection of the real trajectory in this sub-dimensional phase space. But, when this parameter is greater than the real dimension the representation remains accurate, but the computation time and resources increase significantly.

In order to highlight the cavitation phenomenon, we quantify the diagonal line from the distance matrix, as shown in eq. (8) :

$$
s d[n]=\frac{1}{M-n} \sum_{i=1}^{M-n} D\left(v_{i}, v_{i+n}\right)
$$

where $n=\overline{1, M-1}, D\left(v_{i}, v_{i+n}\right)$ are the elements of the $n^{\text {th }}$ diagonal line of the distance matrix considering that the zero diagonal line is the main diagonal of the matrix.

Then, the spectral content for this measure is studied:

$$
S D[k]=\sum_{n=0}^{M-1} s d[n] \cdot \exp \left(-\frac{j 2 \pi k n}{M}\right)
$$

In a very simplified way, we will prove that this measure points out more information than the classical Fourier transform regarding its spectral components.

The most intuitive way to prove the statement above is to consider the following time series:

$$
x[n]=\cos [\Omega n] \cdot \cos [\omega n], \Omega \gg \omega
$$

where $\Omega$ is the parameter characterizing the wide band acoustic signal and $\omega$ is the effect induced by the cavitational vortex.

Considering only a two-dimensional phase space representation and using the squared Euclidean distance, it goes that: 


$$
\begin{gathered}
\overrightarrow{v_{i}}=x[i] \cdot \overrightarrow{e_{1}}+x[i+d] \cdot \overrightarrow{e_{2}} \\
D\left(v_{i}, v_{i+n}\right)=(x[i]-x[i+d])^{2}+(x[i+n]-x[i+n+d])^{2} \\
=E_{i}^{2}[n]+E_{i+d}^{2}[n]
\end{gathered}
$$

Inserting eq. (10) in eq. (12), results in:

$$
E_{i}[n]=2 \alpha \frac{\sin [\Omega(2 i+n)]}{2} \frac{\sin [\omega(2 i+n)]}{2}
$$

where $\alpha=\frac{\sin [(\Omega+\omega) n]}{2} \cong \frac{\sin [(\Omega-\omega) n]}{2}$ when $\Omega \ngtr \omega$.

Hereby, replacing eq. (13) in eq. (12), it gives:

$$
\begin{aligned}
s d[n] \propto D\left(v_{i}, v_{i+n}\right) \cdot \alpha^{-2} & =1+\cos [\omega(2 i+n)]-\cos [\Omega(2 i+n)] \\
& -\cos [\omega(2 i+n)] \cdot \cos [\Omega(2 i+n)]+1+ \\
& +\cos [\omega(2 i+2 d+n)]-\cos [\Omega(2 i+2 d+n)] \\
& -\cos [\omega(2 i+2 d+n)] \cdot \cos [\Omega(2 i+2 d+n)]
\end{aligned}
$$

From eq. (14), it should be noticed that, for the amplitude modulation and/or frequency modulation when $\Omega \gg \omega$, on the amplitude spectrum of the measure $S D$, there are present not only the classical components $\Omega \pm \omega$, but also two extra components appear: $\omega$, respectively, $\Omega$.

This observation will be used for the experimental part, based on the assumption that the effect of the cavitational vortex is highlighted by the lower frequency component $\omega$.

\section{EXPERIMENTAL SETUP AND RESULTS}

The experimental part was performed on a reduced scale facility which consisted of a hydraulic installation where the flow rate can be manually changed [9]. The pipeline of the installation had placed inside a stator which produced the cavitational vortex as the flow rate increased. Fig. 2 presents schematically the experimental configuration.

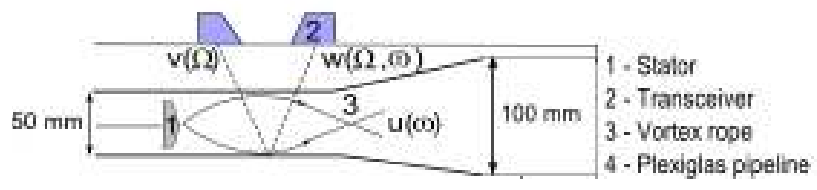

Fig. 2: The experimental configuration

In order to highlight the cavitational vortex presence and to characterize its evolution, the flow rate of the hydraulic system was varied and, when the flow rate became stable, the acoustic active system was started.

In the same time the flow rate of the system was recorded. Moreover, on the tip of the stator a pressure sensor was placed so that we have a reference for the cavitational vortex (the more the pressure is dropping, the higher the intensity of the cavitation is). Fig. 3 presents the setup of the experiment.
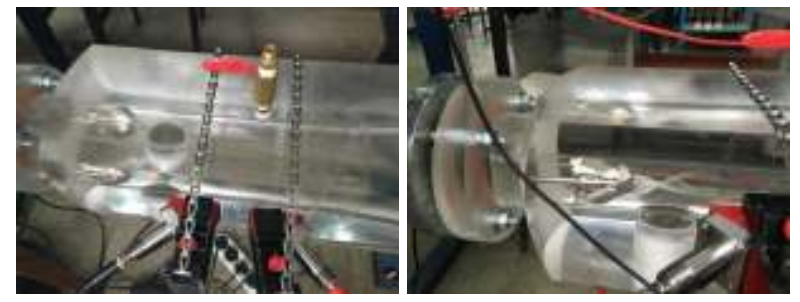

Fig. 3: The on-site setup of the experiment

The acoustic transducers are placed in "V" configuration for a longer interaction with the vortex. The emission transducer, the closest to the stator, generates $1 \mathrm{~ms}$ linear frequency modulations in a spectral bandwidth range of [0.8,1.2] $\mathrm{MHz}$ with a repetition rate of $2.5 \mathrm{~ms}$. The sampling frequency is $5 \mathrm{MHz}$.
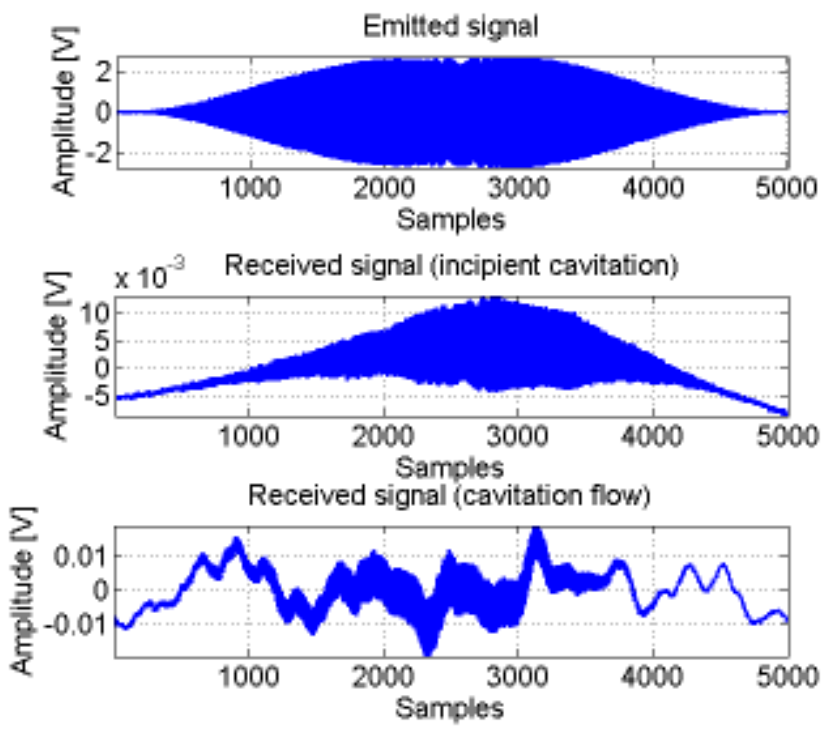

Fig. 4: The emitted signal for each measurement, a received signal during a trial for the incipient cavitation and a received signal during a trial for the cavitation flow

Fig. 4 presents the wide band acoustic signal used for the characterization of the cavitational vortex and two received signals in different states of the system: for the incipient cavitation, respectively, for a fully developed cavitation.

In Fig. 5, the limitations of the Fourier transform applied on the signal's envelope are presented. The signal considered in this case is a signal acquired when the cavitation is clear eye visible (cavitation flow).

This approach is based on the assumption made in section 2 , namely that the spectral content of the cavitation has a much frequency than the wide band acoustic signal of the transceivers $\Omega \approx \omega$. It can be seen that the associated phenomena (attenuation, dispersion, noise, etc.) hide the effect induced by the particle motion from the cavitational vortex. 


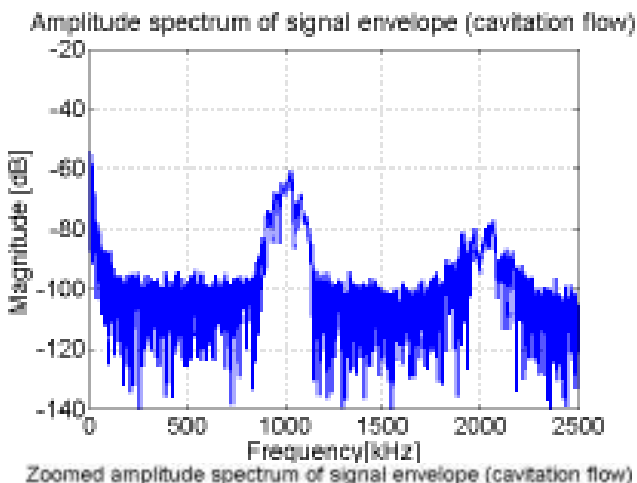

Zoomed amplitude spectrum of signal ervelope (cavtation flow)

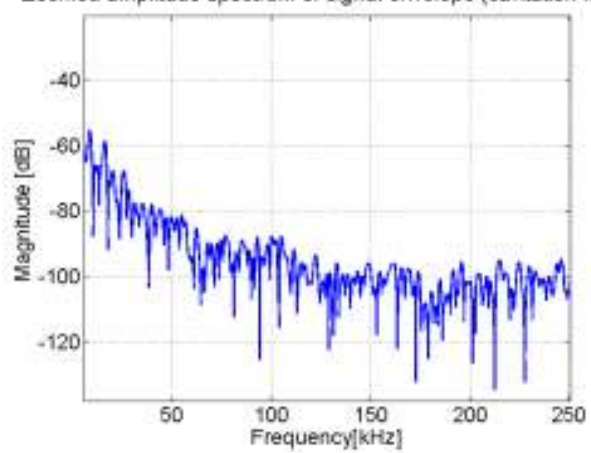

Fig. 5: The amplitude spectrum of the envelope for a received signal (fully developed cavitational vortex)

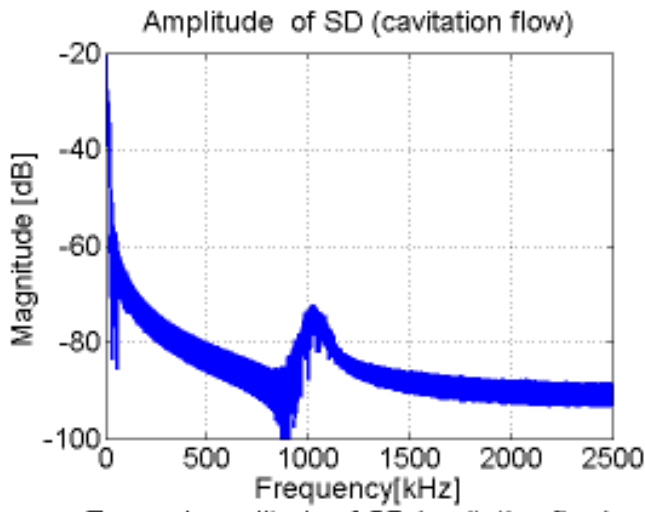

Zoomed amplitude of SD (cavitation flow)

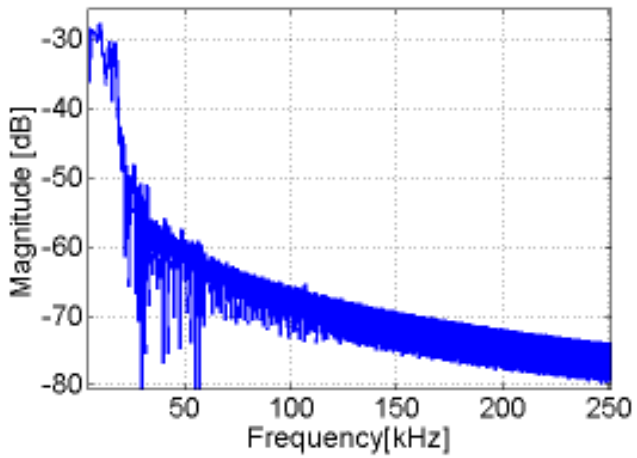

Fig. 6: The amplitude of $S D$ for a received signal (fully developed cavitational vortex)
On the other hand, the use of $S D$ (eq. (9)) highlights, on the same signal, the cavitational vortex spectral content induced in the received signal when the cavitation is fully developed (Fig. 6).

Next, we applied our approach for all acquired signals in each configuration. TABLE 1 presents each configuration and its characterizing parameters.

TABLE 1 CONFIGURATION PARAMETERS

\begin{tabular}{|c|c|c|c|}
\hline \multirow{2}{*}{$\begin{array}{c}\text { Configuration } \\
\text { number }\end{array}$} & $\begin{array}{c}\text { Flow rate } \\
(\boldsymbol{l} / \mathbf{s})\end{array}$ & $\begin{array}{c}\text { Mean } \\
\text { pressure } \\
\text { (bar) }\end{array}$ & $\begin{array}{c}\text { Pressure standard } \\
\text { deviation (Pa) }\end{array}$ \\
\cline { 2 - 4 } & 1.14 & 1.23 & 1936 \\
\hline 3 & 4.34 & 1.19 & 2437 \\
\hline 4 & 9.2 & 1.05 & 3447 \\
\hline 5 & 12.86 & 0.83 & 5784 \\
\hline 6 & 14.77 & 0.64 & 6208 \\
\hline 7 & 15.63 & 0.55 & 6985 \\
\hline 8 & 16.01 & 0.51 & 7136 \\
\hline 9 & 16.08 & 0.49 & 7039 \\
\hline 10 & & & \\
\hline
\end{tabular}

It should be noticed that the greater the number of the configuration, the greater the flow rate of the hydraulic system. This has as a direct consequence the appearance of the cavitation which is emphasized by the decrease of the pressure and the increase of its standard deviation.

\section{SD component for all configuration}

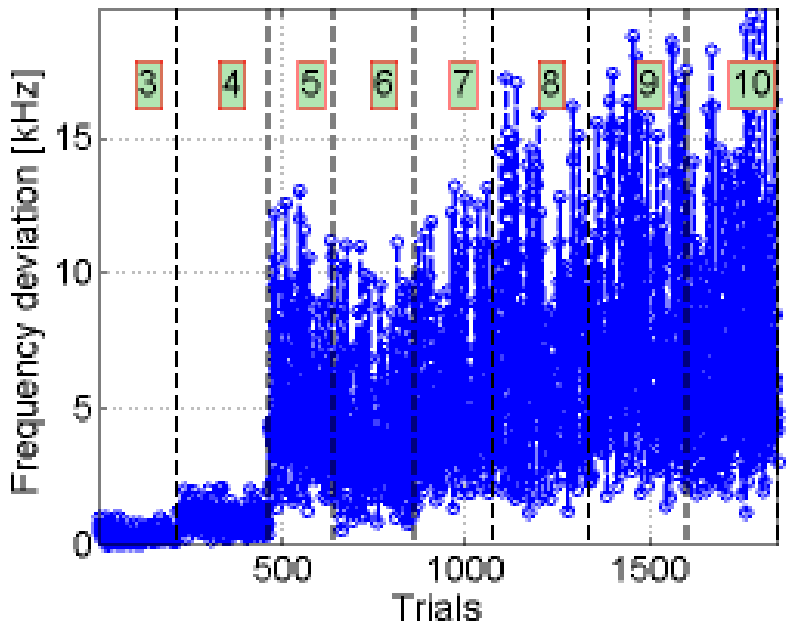

Fig. 7: The frequency deviation using the RPA approach for the signals acquired in each configuration (the number in red box indicates the configuration)

As Fig. 7 presents, in configuration 3, there is no cavitation present. In configuration 4 , the mean of the frequency deviation increases as well as its standard deviation, then, from configuration 5 , the mean and the standard deviation of the frequency component induced by the particle motion increase gradually. 
TABLE 2 ACOUSTIC SIGNAL APPROACH

\begin{tabular}{|c|c|c|}
\hline \multirow[b]{2}{*}{$\begin{array}{c}\text { Configuration } \\
\text { number }\end{array}$} & \multicolumn{2}{|c|}{ Parameters } \\
\hline & $\begin{array}{l}\text { Mean of the } \\
\text { frequency } \\
\text { component (kHz) }\end{array}$ & $\begin{array}{l}\text { Standard deviation of } \\
\text { the frequency } \\
\text { component }(\mathrm{kHz})\end{array}$ \\
\hline 3 & 0.84 & 0.23 \\
\hline 4 & 1.42 & 0.39 \\
\hline 5 & 5.65 & 2.6 \\
\hline 6 & 4.7 & 2.3 \\
\hline 7 & 5.9 & 2.7 \\
\hline 8 & 7.07 & 3.42 \\
\hline 9 & 7.75 & 3.74 \\
\hline 10 & 7.48 & 3.85 \\
\hline
\end{tabular}

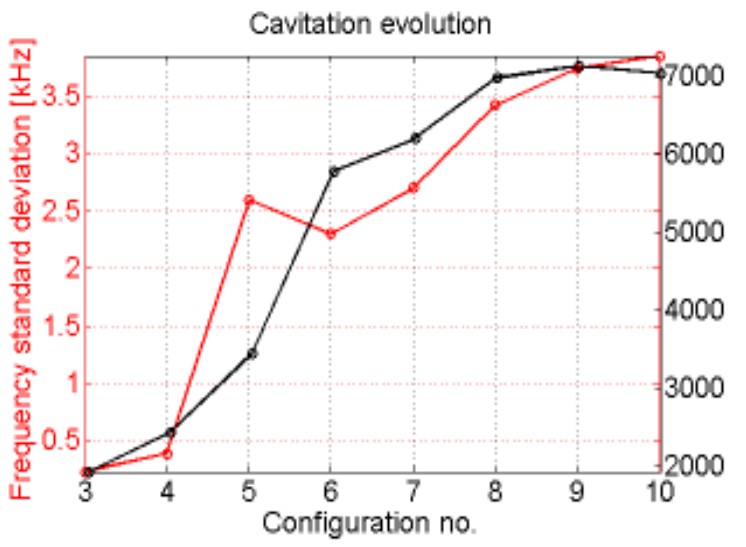

Fig. 8: The cavitation evolution characterized in terms of frequency standard deviation (in red) and pressure standard deviation (in black)

From TABLE 2 and Fig. 8, it can be seen that the mean of the frequency component induced by the particle motion has the same trend as the flow rate of the system, whereas the standard deviation of this component (non-intrusive technique) emphasized the cavitation phenomenon and has the same trend as the pressure standard deviation (intrusive technique).

\section{CONCLUSIONS}

The paper presents an alternative to the characterization of cavitating vortex flow based on a non-intrusive acoustic technique which is easy to set-up on industrial sites.

It is presented the concept of RPA which exploits the physics of the cavitation and not the results, as the imaging techniques do.
The diagonal lines reflect the fundamental frequency of a signal [8]. Based in this fact, we proved that the spectral analysis of these diagonal lines quantification brings into light the effect of an interaction between two different waves, in certain conditions.

We have proven that the cavitating vortex has a spectral component which can be extracted using the diagonal lines quantification. This component describes the evolution of flow and of the cavitating vortex.

Our future work considers confirming the new approach on other applications, such as the rotational vortex of a turbine's draft tube.

\section{ACKNOWLEDGMENT}

The paper part has been funded by the Executive Agency for Higher Education, Research, Development and Innovation, PN-II-PT-PCCA-2013-4, no. 88/2014, ECOTURB project.

This work has also been supported by the SATT Linksium funding in the framework of the MOTRHYS project.

\section{REFERENCES}

[1] W. L. Rubin. "Radar-Acoustic Detection of Aircraft Wake Vortices." Journal of Atmospheric and Oceanic Technology, 2000: pp. 1058-1065.

[2] A. Digulescu, I. Candel, C. Ioana, G. Vasile, G. Dunca, D.M. Bucur, A. Serbanescu, "Water Hammer Effect Characterization using an Acoustic Signal Processing Approach", 2015, 9th International Symposium On Advanced Topics In Electrical Engineering (ATEE)

[3] A. Digulescu, T. Petrut, I. Candel, C. Ioana, F. Bunea, G. Dunca, D.M. Bucur, A. Serbanescu, "Rotational vortex rope detection using recurrence plot analysis", MTA Review; Vol. XXV, No. 1, Mar. 2015;pp. 5-16

[4] N. Takahashi, T. Miyazaki. "The Influence Of Turbulence On A Columnar." Proceedings in Applied Mathematics and Mechanics. 2006.

[5] K.A. Emanuel. "A Note on the Stability of Columnar Vortices." Journal of Fluid Mechanics 145 (1984): pp. 235-238.

[6] N. Marwan, S. Schinkel, J. Kurts. "Recurrence plots 25 years later: Gaining confidence in dynamic transitions." Europhysics Letters, 101, 20007 (2013).

[7] M. Thiel, M.C. Romano, J. Kurths, R. Meucci, E. Allaria, T. Arecchi. "Influence of the observational noise on the recurrence quantification analysis." Phisica D 171 (2002): pp. 138-152.

[8] C. Ioana, A. Digulescu, A. Serbanescu, I. Candel, F.M. Birleanu, "Recent advances in non-stationary signal processing based on Recurrence Plot Analysis concept", Vol. 103, in "Translational Recurrences. From Mathematical Theory to Real-World Applications", pp 75-93. Springer Proceedings in Mathematics \& Statistics, 2014.

[9] I. Candel , F. Bunea, G. Dunca, D.M. Bucur, C. Ioana, B. Reeb, G.D. Ciocan, "Detection of cavitation vortex in hydraulic turbines using acoustic techniques", 27th IAHR Symposium-Hydraulic Machinery and Systems (IAHR 2014), Montreal Canada, 2014. 